\title{
Ethik im Medizincurriculum Wien fest etabliert
}

\author{
Angelika Hofhansl · Anita Rieder · Thomas E. Dorner
}

Eingegangen: 14. November 2014 / Angenommen: 5. Januar 2015 / Online publiziert: 11. März 2015

(C) Springer-Verlag Wien 2015

Sehr geehrte Herausgeber,

Im Artikel „Ethik und ärztliches Ethos im Medizinstudium und im Gesundheitswesen“ [1] haben wir berichtet, wie der Bereich der Medizinethik optimal in einem Medizin Curriculum integriert und vermittelt werden soll. Inzwischen können wir berichten, dass die Medizinethik einen Fixpunkt im vierten Studienjahr des Medizin Curriculum Wiens (MCW) darstellt. Mehrere der im Artikel angesprochenen Themenbereiche sind also im MCW bereits fest etabliert.

Seit dem Wintersemester 2012/13 ist die Medizinethik im vierten Studienjahr im Block 22/23 „Public Health“ im Umfang von zwölf akademischen Stunden (davon sechs als interaktiver Kleingruppenunterricht) verankert und wurde damit im Vergleich $\mathrm{zu}$ früheren Studienjahren verdoppelt [2]. Medizinethik wird dabei neben anderen Themengebieten wie z. B. Public Health [3] interdisziplinär unterrichtet und aus vier verschiedenen Blickwin-

Mag. Dr. A. Hofhansl, MME ( $\varangle)$

Department für Med. Aus- und Weiterbildung, DEMAW

Curriculumkoordination

Medizinische Universität Wien

Spitalgasse 23

1090 Wien, Österreich

E-Mail: angelika.hofhansl@meduniwien.ac.at

Univ.Prof. Dr. A. Rieder

Curriculumdirektorin Humanmedizin

Institut für Sozialmedizin, Zentrum für Public Health

Medizinische Universität Wien

Kinderspitalgasse 15

1090 Wien, Österreich

E-Mail: anita.rieder@meduniwien.ac.at

Assoc.Prof. Priv.Doz. Dr. T. E. Dorner, MPH

Institut für Sozialmedizin, Zentrum für Public Health

Medizinische Universität Wien

Kinderspitalgasse 15

1090 Wien, Österreich

E-Mail: thomas.dorner@meduniwien.ac.at keln beleuchtet: Ethik im Gesundheitssystem, Ethische Aspekte in der Arzt-Patient Beziehung, Ethische Fragen bei sterbenden Patientinnen und Patienten/Palliativmedizin, Ethnomedizin sowie Ethik und Interkulturalität. Für alle diese Bereiche wurden von den Themenverantwortlichen konkrete Lernziele im Studienführer („Study Guide") für die Studierenden formuliert [4]. Neben Lernzielen auf der Wissensebene (Knowledge) sind dies auch Lernziele auf dem viel anspruchsvolleren Level der Haltungen und Einstellungen (Attitudes).

Im Thema „Ethik im Gesundheitssystem“ erwerben die Studierenden theoretischen Grundlagen zum Thema Medizinethik und deren Schnittstellen im Gesundheitswesen. Darauf aufbauend werden sie für das Empfinden von Gerechtigkeit und Ungerechtigkeit sensibilisiert.

Im Thema „Ethische Aspekte in der Arzt-Patient Beziehung" werden wesentliche Aspekte der Selbstbestimmung - sowohl auf Seiten der Patientinnen und Patienten als auch auf Seiten der therapeutischen Partnerinnen und Partner - erarbeitet. Basierend darauf wird der Aufklärungsprozess vermittelt.

Im Thema „Ethisches ärztliches Handeln“ lernen die Studierenden, wie man den ethischen Diskurs auf der sach- und wertorientierten Ebene führt.

Im Thema „Ethische Fragen bei sterbenden Patientinnen und Patienten/Palliativmedizin" werden wesentliche Unterschiede hinsichtlich der ärztlichen Sterbehilfe und -begleitung diskutiert und aus verschiedenen ethischen Blickwinkeln beleuchtet.

Im Thema Ethnomedizin, Ethik und Interkulturalität werden interkulturelle Dimensionen von Ethik, Krankheit/Kranksein sowie von Alter, Sterben und Tod vorgestellt und diskutiert. Dabei geht es sowohl um die Vermittlung von spezifischem kulturellem Wissen als auch um die Reflexion von Einstellungen und Haltungen im klinischen Alltag. Ziel ist es, die Studierenden auf die ethnische und kulturelle Diversität von Patientinnen und Patienten zu sensibilisieren. Ein besonderes Augenmerk 
liegt dabei auf der speziellen Situation vulnerabler Gruppen, wie z. B. Migrantinnen und Migranten oder Personen, die einer Minderheit angehören.

Zusätzlich zu diesen speziell als „Medizinethik“ ausgewiesenen Lehrveranstaltungen gibt es im Block 22/23 „Public Health“ weitere Lehrveranstaltungen, in denen ethische Aspekte thematisiert und diskutiert werden. So wird den Studierenden etwa im Seminar „Fehlermanagement" vermittelt, dass Fehler zum menschlichen Handeln dazugehören und nie gänzlich vermeidbar sind. Den Studierenden wird eine Fehlerkultur vorgestellt, in der es nicht darum geht, Fehler zu vertuschen, sondern Fehler stets als ein „Window of Opportunity“ zu begreifen. Ziel ist es, aus Fehlern zu lernen - sowohl auf persönlicher Ebene als auch auf Ebene des Systems, in dem sie passieren.

Eines der expliziten Lernziele im Seminar „Schmerz: Soziale, ökonomische und versorgungsbezogen Aspekte“ ist es, dass Patientinnen und Patienten mit chronischen Krankheiten und sogenannten „durchgemachten Patientinnen- und Patientenkarrieren“ stets ernst genommen und in ihrem Selbstmanagement der Gesundheitsbeeinträchtigung unterstützt werden müssen. Voraussetzung für diese Kompetenz ist ein Selbstverständnis der ArztPatient Beziehung auf gleicher Augenhöhe.

Alle oben angeführten Themenbereiche sind auch Gegenstand von standardisierten Prüfungsmethoden im MCW. So kann sichergestellt werden, dass die intendierten Lernziele von den Studierenden auch erreicht werden.

Die Verankerung dieser Themenbereiche der Medizinethik in das Medizin Curriculum Wien spiegelt sich auch in internationalen Empfehlungen [5] wider. Evaluationen zeigen, dass die Studierenden diese Inhalte, welche nicht direkt auf kurative Medizin ausgerichtet sind, sehr gut aufnehmen. Das belegt beispielsweise eine Untersuchung an Studierenden des MCW zu Einstellungen, Fertigkeiten und Wissen bezüglich Gesundheitsförderung und Präventivmedizin [6].

\section{Interessenkonflikt}

A. Hofhansl, A. Rieder und T.E. Dorner geben an, dass kein Interessenskonflikt besteht.

\section{Literatur}

1. Körtner U, Hofhansl A, Dinges S. Ethik und ärztliches Ethos im Medizinstudium und im Gesundheitswesen. Wien Med Wochenschr. 2014;164: 34-41.

2. Dorner TE. (Hrsg.) Public Health. Von den Gesundheitsbedürfnissen der Gesellschaft zu klinischen Implikationen. Facultas Verlag, Wien. ISBN 978-3-7089-1021-5; 2. Aufl.; 2014.

3. Diem G, Dorner TE. Public Health Ausbildung in Österreich. Eine Übersicht. Wien Med Wochenschr. 2014;164:131-40.

4. https://studyguide.meduniwien.ac.at/curriculum/n202$2014 /$ ?state $=0-65349-3725 /$ kapitel-3-medizinethik. Zugegriffen: 30. Oct. 2014.

5. Goldie J. Review of ethics curricula in undergraduate medical education. Med Educ. 2000;34:108-19.

6. Borsoi L, Rieder A, Stein KV, Hofhansl A, Dorner TE. Preventive medicine: self-assessment of knowledge, skills and attitudes of medical students at the Medical University of Vienna. Wien Med Wochenschr. 2014;164:146-51. 